\title{
A simple, robust and semi-automated parasite egg isolation protocol
}

\author{
Ted HM Mes ${ }^{1}$, Maarten Eysker ${ }^{2}$ \& Harm W Ploeger $^{2}$

\begin{abstract}
${ }^{1}$ Netherlands Institute of Ecology (NIOO-KNAW), Centre for Estuarine and Marine Ecology, POB 140, 4400AC, Yerseke, The Netherlands. ${ }^{2}$ Department of Infectious Diseases and Immunology, Faculty of Veterinary Medicine, Utrecht University, POB 80.165, 3508 TD Utrecht, The Netherlands. Correspondence should be addressed to T.H.M. (t.mes@nioo.knaw.nl).
\end{abstract}

Published online 15 March 2007; doi:10.1038/nprot.2007.56

\begin{abstract}
Large-scale parasite quantification is required for improving our understanding of the epidemiology and genetics of host-parasite interactions. We describe a protocol that uses a low-density salt solution for flotation and centrifugation of nematode eggs. Subsequently, sucrose flotation and precipitation are used to obtain clear egg preparations. Most traditional quantification protocols such as the McMaster technique are unsuited for the standardized processing of large numbers of samples and the analysis of large amounts of feces per sample. Consequently, they are suited only for small-scale surveys. Our protocol, which can be used to analyze up to $6 \mathrm{~g}$ of feces, results in clear egg preparations that are concentrated in wells of a microtiter plate and that are suited for digital recording and automated counting. Starting from a fecal suspension in the first flotation solution to a digital recording requires approximately $40 \mathrm{~min}$ per 24 samples.
\end{abstract}

\section{INTRODUCTION}

The concentration of parasites, most notably the number of parasite eggs, is an important parameter in epidemiology, studies of drug resistance, the efficacy of biological control of nematode parasitism and the diagnosis of infections. Obtaining parasite egg counts (EPG; eggs per gram) is relatively straightforward; essentially unchanged protocols have existed for decades, such as the McMaster technique $^{1}$ and the Wisconsin flotation technique ${ }^{2}$. Typically, feces are homogenized in dense salt or sugar solutions, followed by collection of the eggs in counting chambers ${ }^{1}$ or by adherence of eggs to microscope coverslips after centrifugation ${ }^{2}$. These methods require instantaneous and manual counting of eggs $^{1,2}$ and can be expected to introduce considerable variation in replicate experiments ${ }^{2}$. Only relatively small amounts of feces can be analyzed, which hampers large-scale studies of parasite transmission and resistance, owing to time and resource issues. One of the commonly used techniques, the McMaster technique, has a theoretical lower detection limit of $50 \mathrm{EPG}^{1}$, which is inadequate to effectively screen resistant and/or immune animals. Collectively, these issues seriously compromise modern approaches that use epidemiologic and genetic inferences as an aid to the management of the economic and societal costs of parasitic diseases.

Here, we present a robust method that can be used to obtain clear preparations of nematode eggs from feces from either artificially or naturally infected sources ${ }^{3}$. This nematode egg isolation technique, referred to as the salt-sugar flotation (SSF) method, allows large numbers of samples to be processed due to three factors. First, the handling and processing of samples is simple, requires little effort and can be expected to introduce less technical variation than other protocols (e.g., ref. 2). Second, the eggs are concentrated in a very small area $\left(\approx 1 \mathrm{~mm}^{2}\right)$, which allows digital recordings to be made. This is important because counting nematode eggs is laborious and time-consuming. Moreover, given the seasonal workload typical when studying parasites, it is definitely advantageous that egg concentrations need not be analyzed instantaneously. It has traditionally been problematic to automatically separate and/or distinguish small objects such as straws and air bubbles from eggs on digital images, in part due to low levels of contrast. Using the egg isolation protocol described here, the image analysis software readily distinguishes these objects in clear egg preparations based on gray scale, shape and size attributes to eliminate this problem. Finally, the amount of feces that can be analyzed is much larger than that in traditional methods $s^{1,2,4,5}$. For example, we routinely analyze $3 \mathrm{~g}$ of feces (with a lower detection limit of 0.33 EPG, 150 times lower than the standard McMaster technique, $\mathrm{cf}^{1}$ ). This difference is due to the fact that only a small portion of the $3 \mathrm{~g}$ of feces used in the McMaster technique is analyzed. Our protocol can also be scaled upwards to analyze even larger amounts (up to $6 \mathrm{~g}$ ). This is expected to lead to more sensitive and precise measurements compared to traditional methods. In particular, the increased precision and sensitivity of this method can be valuable for studies of animals with low EPGs such as adult cows. In large-scale surveys of the Dutch dairy cattle population, we found a substantially higher proportion of positive adult and resistant animals than the traditional egg isolation methods. Specifically, the proportion of negative animals decreased from $>70 \%$, using a modified form of the McMaster egg isolation method $^{4,5}$, to $21 \%$ (see ref. 3 ).

Using this protocol, we detected eggs of seven species of gastrointestinal nematode species of livestock, which have an impact on the productivity and efficacy of the ruminant industry (Cooperia oncophora, C. punctata, Ostertagia ostertagi, Trichostrongylus sp, Haemonchus sp, Oesophagostomum sp and Bunostomum sp). It is likely that the eggs of other helminths can be isolated using this protocol, because many of these are also currently isolated using flotation techniques. These include plant parasitic nematodes that are usually detected using filters and stains ${ }^{6}$. It is possible that the gravity of the flotation solutions may need adjustment, which can be examined under a microscope. Densities of sugar flotation solutions have also been reported for some important helminth species $^{7}$, which suggests that the SSF protocol is applicable to nematode and trematode parasites from plants, animals and 
humans. It is possible that the heavy trematode eggs can also be isolated using the SSF protocol, although this might require denser flotation solutions than those used here.

There are also some limitations and drawbacks to the SSF protocol. One is the requirement for centrifugation. The number of samples that can be processed in parallel depends strongly on the type of centrifuge that is available. The same constraint applies to sample volumes. In addition, egg preparations are occasionally contaminated with mite eggs. These, however, are easily distinguishable from trichostrongylid eggs (not shown), and they are not recognized as nematode eggs by the image analysis software.

\author{
MATERIALS \\ REAGENTS \\ - $\mathrm{NaCl}$ (Sigma-Aldrich, S7653) \\ - Sucrose (Sigma-Aldrich, S7903) \\ EQUIPMENT \\ - Coarse metal sieve approximately $5 \mathrm{~cm}$ diameter (pore size $4 \mathrm{~mm}^{2}$ ) and flask \\ of approximately $6-6.5 \mathrm{~cm}$ diameter and $21 \mathrm{~cm}$ height \\ - Benchtop centrifuge $\Delta$ CRITICAL Provide at least 3,600 $\mathrm{g}$ at room temperature. \\ - Digital camera with zoom objective that can be fitted onto a microscope \\ - Microscope \\ - Pipette $(200 \mu \mathrm{l})$ \\ -24- or 96-well microtiter plates \\ - Mineral oil $\Delta$ CRITICAL Low quality is fine.
}

\author{
$\cdot 15 \mathrm{ml}$ centrifuge tubes \\ - $50 \mathrm{ml}$ Falcon tubes \\ -Weighing scale $\Delta$ CRITICAL Should be of at least $0.1 \mathrm{~g}$ accuracy. \\ -Water vacuum pump \\ REAGENT SETUP \\ $\cdot 13 \%(\mathrm{w} / \mathrm{v}) \mathrm{NaCl}$ solution (specific gravity 1.06 ) \\ $\cdot 17 \%(\mathrm{w} / \mathrm{v})$ sucrose solution (specific gravity 1.06$)$ \\ $\cdot 70 \%(\mathrm{w} / \mathrm{v})$ sucrose solution (specific gravity 1.27 ) \\ EQUIPMENT SETUP \\ Digital camera attached to binocular microscope $\triangle$ CRITICAL We used a \\ Coolpix camera attached to a Nikon microscope at 10 or 30 times \\ magnification. Other types of digital camera may also be used.
}

\section{PROCEDURE}

Egg isolation

1) Weigh up to $6 \mathrm{~g}$ of feces in a $50 \mathrm{ml}$ Falcon tube. Add $25 \mathrm{ml}$ of $13 \% \mathrm{NaCl}$ solution and shake vigorously until all fecal matter is suspended, to liberate eggs from feces.

$\triangle$ CRITICAL STEP Larger amounts of feces may be used, but this requires larger volumes of the salt solution and, consequently, larger centrifuge tubes. We primarily applied the method to feces of dairy cattle, which is relatively easy to homogenize. Feces of other hosts such as sheep have to be shaken much longer.

2| Pour the suspension over the coarse metal sieve to separate eggs from fecal matter and other debris, and collect the egg-containing through-flow in a flask.

3| Pour the through-flow into a $50 \mathrm{ml}$ Falcon tube and centrifuge at $3,600 \mathrm{~g}$ at room temperature (RT; $15-30{ }^{\circ} \mathrm{C}$ is suitable) for $5 \mathrm{~min}$. The nematode eggs will float to the top of the flotation solution.

4| Pour the egg-containing supernatant into another $50 \mathrm{ml}$ Falcon tube and add equal volume of tapwater to the supernatant. $\triangle$ CRITICAL STEP The amounts of feces and volumes of the $\mathrm{NaCl}$ and sucrose solutions can be changed (according to the centrifuge capacity, bucket size or the anticipated concentration of eggs, for example). However, the addition of an equal volume of tapwater needs to be adhered to. Failure to do so may prevent eggs from being precipitated, leading to a loss of eggs.

5| Mix and centrifuge again for $1 \mathrm{~min}$ at 3,600 $\mathrm{g}$ at RT. The nematode eggs will sink to the bottom of the centrifuge tube.

6| Remove the supernatant with a water pump and resuspend the egg-containing pellet in $5 \mathrm{ml}$ of $17 \%$ sucrose.

$\triangle$ CRITICAL STEP At this point, it is possible to downscale the volume of egg preparation, by using smaller tubes that are appropriate for the processing of larger numbers of samples and changing the volume of the $17 \%$ sucrose solution to be added (this can be changed as desired, if the volume of the salt solution above the egg pellet is small. In $15 \mathrm{ml}$ centrifuge tubes, the volume of the egg suspension can be reduced to approximately $200 \mu \mathrm{l}$. When using such a small volume for egg preparations, the volume of sucrose solution added can safely be reduced to $2 \mathrm{ml}$ ). We use 15 or $50 \mathrm{ml}$ Falcon centrifuge tubes, but smaller tubes, including $1.5 \mathrm{ml}$ Eppendorf tubes, may be used. Although the salt and sucrose flotation solutions have the same specific gravity, we use the sucrose solution at this step, because it is more viscous. This makes it easier to take digital images. The salt solution used for flotation is most convenient for homogenization of feces.

7| Centrifuge at 3,600 g at RT for 5 min. The nematode eggs will float to the top of the flotation solution.

8| Transfer the egg-containing supernatant to a new tube of appropriate size and dilute once with an equal volume of tapwater.

9| Mix thoroughly (e.g., on a vortex) and centrifuge at 3,600 g at RT for $5 \mathrm{~min}$. The nematode eggs will sink to the bottom of the centrifuge tube. 
$\triangle$ CRITICAL STEP Optionally, this second precipitation-flotation (Steps 6-9) can be discarded if there is no need to increase the clarity of egg preparations. This may be applicable if egg concentrations are sufficiently high so that small amounts of feces can be used, or if eggs of plant parasitic nematodes are isolated.

10| Remove the supernatant by gently reducing the volume of the egg-containing solution to approximately $200 \mu$ using, for example, a waterpump.

$\triangle$ CRITICAL STEP The eggs are on the bottom of the centrifuge tubes. Make sure that they are not removed. Usually, removing the flotation solution until $1 \mathrm{~cm}$ from the bottom of the tube is safe.

11 Add three drops of $70 \%$ (saturated) sucrose solution. Mix the suspension vigorously by repeated pipetting of the solution or vortexing.

\section{Egg counting and imaging}

12| Egg counting and imaging can be accomplished either manually (option A) or automatically (option B).

(A) Manual egg counting and imaging

(i) Fill 96-well microtiter plates with three drops of mineral oil.

(ii) Transfer samples from Step 11 onto the plate.

$\triangle$ CRITICAL STEP Transfer underneath the oil to ensure a positive meniscus.

(iii) Allow samples to settle for 15 min; this allows eggs to concentrate at the top of the meniscus.

- PAUSE POINT Eggs remain undamaged for at least $4 \mathrm{~h}$. However, much longer storage will damage the eggs

(e.g., storage longer than $24 \mathrm{~h}$ will cause the eggs to get diffuse due to disintegration).

(iv) Scan the egg suspensions with a Coolpix camera (Nikon) attached to a Nikon binocular microscope (SMZ800) at $\times 30$ magnification.

(v) Save pictures in JPEG format (file size approximately 350-400 kb) and count eggs manually. In case of manual countings, all eggs are counted.

\section{(B) Automatic egg counting and imaging}

(i) Transfer samples from Step 11 onto 24-well plates, without adding mineral oil.

(ii) Add a corresponding volume of saturated sucrose solution, depending on the well volume. The final sucrose concentration should be $17 \%$ or higher. We use a total volume of approximately $2 \mathrm{ml}$.

(iii) Allow samples to settle for 15 min.

- PAUSE POINT Eggs remain undamaged for at least $4 \mathrm{~h}$. However, much longer storage will damage the eggs (e.g., storage longer than $24 \mathrm{~h}$ will cause the eggs to get diffuse due to disintegration).

(iv) Scan the egg suspensions with a Coolpix camera (Nikon) attached to a Nikon binocular microscope (SMZ800) at $\times 10$ magnification.

(v) Import the images into a image analysis package (we used the KS 400 system of Carl Zeiss Vision, Germany), in which a dynamic discrimination procedure uses the local neighborhood gray values as a criterion to identify nematode eggs.

(vi) Use the following identifiers to count parasite eggs automatically. Because parasite eggs are of rather constant size and shape, objects of other size and/or shape can be excluded from the counting procedure using the following settings. At $\times 30$ magnification, objects smaller than 40 pixels and larger than 230 pixels are rejected. Objects with a shape parameter, the ratio of the smallest and largest diameter, below 0.39 , such as small straws, and above 0.93 , such as air bubbles, are also rejected. These criteria detect virtually all parasitic nematode eggs (see Fig. 1). In the case of automated counts, a representative piece of the image is counted, the size of which depends on the number of eggs on an image. When using a $\times 10$ magnification and 24-well plates, approximately one-fourth of the surface of a well is scanned. The spatial homogeneity of the egg distribution and the appropriate size of the portion of a well that needs to be scanned will vary from parasite to parasite.

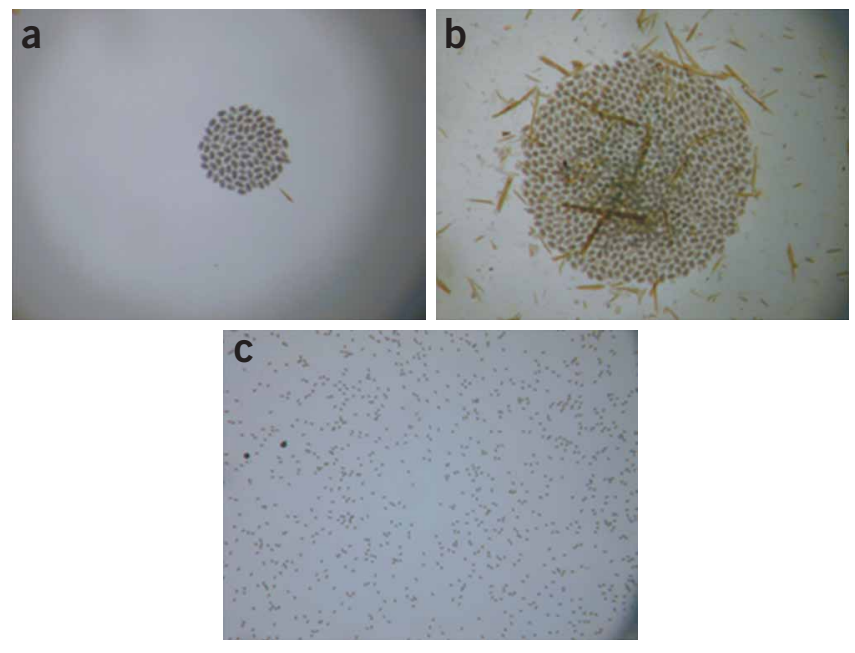

Figure 1 | Examples of egg preparations from the egg isolation protocol. $(\mathbf{a}, \mathbf{b})$ Egg suspensions in 96-well microtiter plates for manual counting, viewed at $\times 30$ magnification. Images show preparations with differing levels of contaminants (e.g., straws). The addition of mineral oil to the 96 -well plate for manual counting results in a positive meniscus, which causes a circular grouping or clumping (but not overlaying) of parasite eggs. (c) Egg preparation for automated counting at $\times 10$ magnification. Large numbers of eggs are spread out, as mineral oil is not used for automated counting and thus there is no meniscus. 
Figure 2 | Characteristics of the SSF method. (a) Correlation between the observed number of parasite eggs detected using the automated egg counting algorithm and the expected number of eggs as determined visually from the images or under the microscope. The regression equation is $y=1.025 x+0.112$. (b) Correlation between the observed and expected EPGs detected by the SSF method using manual egg counts. Egg-free feces were spiked with known numbers of nematode eggs. The regression equation is $y=0.516 x-2.585$.

$\triangle$ CRITICAL STEP In case of extremely high numbers of eggs in the image (several thousands), the eggs aggregate and it is not possible to identify a representative section of a well. Therefore, if very high EPGs are anticipated, such as when dealing with samples from artificial infections, the amount of feces used for egg isolation should be reduced.

\section{TIMING}

Equipment and reagent setup: 0.5 day

Steps 1-12 for manual counts: 1 day (depending on the number of samples). Up to 100 samples can be processed per day Steps 1-12 for automated counts: 1 day. Automatic counting of samples requires only a couple of seconds per sample. The image analysis does not limit the number of samples that can be processed

\section{ANTICIPATED RESULTS}

Following the above protocol, the $\times 10$ magnification shows very low numbers of objects other than parasite eggs. Although the degree of contamination depends on environmental conditions and food, large-scale studies conducted in our laboratory indicate that the vast majority of samples result in clear egg preparation that can easily be analyzed using the image analysis software. Typical examples of digital recordings of egg preparations at $\times 10$ and $\times 30$ magnification are shown in Figure 1 . These different levels of magnification allow samples with egg concentrations of different orders of magnitude to be analyzed (e.g., most artificially and naturally infected samples). As noted above, dense egg preparations may clump. Under these conditions, it is not possible to count a representative section of a well, and automated discrimination of individual eggs may be compromised. This is because egg aggregates have shapes quite different from those of individual eggs. In practice, the range of EPGs that lead to clumping are observed only in artificial infections.

It should be noted that estimates of parasitological burden are almost without exception variable. This is due to parasite- as well as host-related phenomena and the technical variability in egg counting procedures. When using filtering and centrifugation steps for egg isolation, a certain level of variability is unavoidable, although a good correlation between observed and expected egg concentrations was found (Fig. 2a,b). These experiments are based on the number of eggs that were visible on digital images versus those that were counted by the image analysis software (Fig. 2a). Egg-free feces that were spiked with known numbers of eggs also gave a good correlation with the isolated number of eggs (Fig. $\mathbf{2 b}$ ). If there are many thousands of eggs in an egg preparation, the relationship observed in Figure $\mathbf{2} \mathbf{b}$ no longer holds due to the aggregation of eggs and the amount of feces needs to be reduced.

ACKNOWLEDGMENTS We thank M. Terlou, F.N.J. Kooyman and M.P.J. van der Ploeg for help in developing and applying this parasite egg isolation technique. This is publication 3991 of NI00-KNAW. Figure 2 has been reproduced from Mes, Ploeger, Terlou, Kooyman, van der Ploeg and Eysker. A novel method for the isolation of gastro-intestinal nematodeo eggs that allows automated analysis of digital images of egg preparations and high throughput screening. Parasitology 123, 309-314 (2001). Printed with permission from Cambridge University Press.

AUTHOR CONTRIBUTIONS T.H.M.M., design of isolation method and editing of text. M.E., application of method. H.W.P., preparation of figures and editing of text.

COMPETING INTERESTS STATEMENT The authors declare that they have no competing financial interests.

Published online at http://www.natureprotocols.com

Reprints and permissions information is available online at http://npg.nature.com/ reprintsandpermissions
1. MAFF. Manual of Veterinary Parasitological Laboratory Techniques. Technical Bulletin 18. (Freeman and Company, New York, 1977).

2. Cox, D.D. \& Todd, A.C. Survey of gastro-intestinal parasitism in Wisconsin dairy cattle. J. Am. Vet. Med. Ass. 141, 706-709 (1962).

3. Mes, T.H.M. et al. A novel method for the isolation of gastro-intestinal nematode eggs that allows automated analysis of digital images of egg preparations and high throughput screening. Parasitology 123, 309-314 (2001).

4. Agneessens, J., Claerebout, E., Dorny, P., Borgsteede, F.H.M. \& Vercruysse, J. Nematode parasitism in adult dairy cows in Belgium. Vet. Parasitol. 90, 83-92 (2000).

5. Borgsteede, F.H.M., Tibben, J., Cornelissen, J.B.W.J.J., Agneessens, J. \& Gaasenbeek, C.P.H. Nematode parasites of adult dairy cattle in The Netherlands. Vet. Parasitol. 89, 287-296 (2000).

6. Niblack, T.L., Heinz, R.D., Smith, G.S. \& Donald, P.A. Distribution, density, and diversity of Heterodera glycines in Missouri. J. Nematol. 25, 880-886 (1993).

7. David, E.D. \& Lindquist, W.D. Determination of the specific gravity of certain helminth eggs using sucrose density gradient centrifugation. J. Parasitol. 68, 916-919 (1982). 